\title{
Modeling, Simulation and Control of Bioreactors Process Parameters - Remote Experimentation Approach
}

\author{
Jagadeesh Chandra A.P \\ JSS Research Foundation \\ JSS Technical Institutions Campus \\ Mysore
}

\author{
R.D Sudhaker Samuel \\ Sri Jayachamarajendra College of Engineering \\ JSS Technical Institutions Campus \\ Mysore
}

\begin{abstract}
Virtual Instruments are the motivational component for remote experimentation in Science and Engineering. The paper presents the use of Virtual Instruments in simulation and control of bioreactors process parameters, which leads easy extension to remote laboratory practices. The process systems equivalent to bioreactors process parameters, temperature, $\mathrm{pH}$ and agitation speed are modeled and the corresponding control algorithms are developed to obtain the acceptable output response. The sensors and actuators are simulated and the interface to the actual hardware can be established through the data acquisition systems. The graphical user interface developed using LabVIEW transfer information to the networked clients through web server and standard internet browsers for data access.
\end{abstract}

\section{Categories and Subject Descriptors}

J.2 [Computer Applications]: Physical Sciences and Engineering - electronics, engineering.

\section{General Terms}

Algorithms, Measurement, Design, Experimentation.

\section{Keywords}

PID control, Remote Access, Simulation. Virtual Instruments.

\section{INTRODUCTION}

Science and Engineering courses typically involve learners in practical experiments and assignments as part of their knowledge and skill development. Automation is changing the nature of the laboratories and the system designer's focus on the availability of several tools to integrate electronic and mechanical hardware with the networked computing technologies. Internet technologies offer integration capabilities for educational applications both in theoretical as well as laboratory experimentation. With the advent of IT and collaborative learning tools, the remote experimentations are possible, where the learners can access the laboratory set-up from anywhere in the world by connecting to Internet [10]. Web based experiments have been developed in many domains includes, electric circuits, control Systems, microcontrollers, power electronics etc, [3] [6] [8] [11]. The important component of these remote experiments is the VI tools integrated with the real hardware to perform tasks in real time. The supervisory control software and MATLAB for data analysis and visualization is used in process operations and can be networked through OPC (Object Linking and Embedding for process control) based client server communication [7]. Optimal controller design is possible with VI tools and can be used for the real hardware with minor changes in the algorithm by observing the system performance [5]. Making use of advances in technology is an efficient and effective approach in laboratory based course delivery systems.

The control algorithms and techniques described in this paper have two different aspects of usage. One of the aspects is it can be used for educational purposes in teaching and learning of control systems and the other aspect is that it can also be extended to remote experimentation by the control of real systems using data acquisition and signal conditioning circuits to interface with physical components. The control algorithms are developed using Virtual Instruments (VI) and the systems are modeled with the corresponding transfer functions. The control algorithms can be developed using LabVIEW either using basic built in functions [1] or by using control simulation module, but the simulation module provides rich features and easy development of control algorithms and system models [15]. Bioreactors process system is modeled by considering the real specifications of the physical system and control algorithms are developed for the parameters such as temperature, $\mathrm{pH}$ and agitation speed. The remote access features of LabVIEW can be used to integrate all control and measure features of VI with World Wide Web. The complete control environment is created using control simulation module of LabVIEW with appropriately choosing the transfer function models for each control parameter.

\section{PROTOTYPE OF PROCESS SYSTEMS}

The process system is mathematically modeled with suitable transfer function and is derived by considering the specifications and its functionality. The three system parameters considered are temperature, $\mathrm{pH}$ and agitation speed and the corresponding physical components are heater/cooler, pumps/valves and motor are modeled to verify its functionality and stability to obtain tuning coefficients of the control algorithm.

\section{Temperature System}

In many applications such as electrical heating systems, the heating element is controlled by a contactor that has only two states (on or off) [12]. Electric heater controls the temperature of the liquid in a tank and the aim is to control the temperature at the desired value. Most of the temperature control systems can be approximated to a single order transfer function with a transportation delay and time constant which depends on the 
parameters such a specific heat coefficient, temperature coefficient etc., [13].

Transfer function of the temperature system where a liquid at temperature $\theta$ enters a tank which is heated with an electric heater and $\mathrm{q}$ is the heat flow of the heating element with flow rate constant $\mathrm{k}$, specific heat coefficient $\mathrm{P}$, thermal resistance of tank insulation $\mathrm{R}$ and thermal capacitance of water $\mathrm{C}$, is given by,

$$
\begin{aligned}
& \frac{\theta(s)}{q(s)}=\frac{b}{s+a b}, \\
& \text { Where } \quad a=k P+\frac{1}{R}, \quad b=\frac{1}{C}
\end{aligned}
$$

According to Ziegler and Nichols, an open-loop process can be approximated by the transfer function,

$$
G_{T}(s)=\frac{K \exp \left(-s T_{d}\right)}{1+s T_{i}}
$$

The coefficients $K, T_{d}$ and $T_{i}$ are found from a simple open-loop unit step response of the process.

Assuming the process system step response with 30s initial delay and it takes 230 s to rise from $10^{\circ}$ to $60^{\circ}$, then the parameters are calculated as, $K=50, T_{d}=30 s, T_{i}=230 \mathrm{~s}$ and substituting in equation (2),

$$
G_{T}(s)=\frac{50 \exp (-30 s)}{1+230 s}
$$

Equation (3) defines the mathematical model for heating system which controls the temperature of the bioreactors process and suitable control coefficients needs to be calculated for stable operation of the system.

\section{Motor Modeling}

In process control applications, the stirring and agitation are controlled by the motor and a model based on its specifications needs to be obtained. The system is modeled on permanent magnet DC motor and the transfer function, which is the ratio of output angular velocity $\omega(\mathrm{s})$ and the input voltage $\mathrm{v}(\mathrm{s})$ is given by,

$$
G_{M}(s)=\frac{\omega(s)}{v(s)}=\frac{K}{J L s^{2}+(J R+b L) s+\left(R b+K^{2}\right)}
$$

Where the motor constants $\mathrm{K}$ is torque constant, $\mathrm{J}$ is moment of inertia, $\mathrm{R}$ is armature resistance, $\mathrm{L}$ is armature inductance, $\mathrm{b}$ is damping coefficient.

Assuming the motor specifications, $\mathrm{DC}$ voltage $\mathrm{V}=12 \mathrm{~V}$, Power $\mathrm{P}=80 \mathrm{~W}$, Maximum speed $\mathrm{N}=1000 \mathrm{rpm}$, rotor inertia $\mathrm{J}=0.01$, $\mathrm{R}=1 \Omega$ and $\mathrm{L}=0.5 \mathrm{H}$ the other constants are $\mathrm{K}$ and $\mathrm{b}$ are found to be 0.1146 and 0.0073 respectively [2]. Substituting these values in equation (4), the final transfer function of motor becomes,

$$
G_{M}(s)=\frac{0.1146}{0.005 s^{2}+0.01365 s+0.0204}
$$

Equation (5) represents the mathematical model of motor and the feedback control mechanisms have to be designed to maintain the desired speed in industrial process applications.

\section{GRAPHICAL CONTROL ALGORITHMS}

The control algorithms for the process systems are developed using VI tools and the feedback control loop is described in Figure 1. The objective is to understand the process operation and control the process parameters: temperature, $\mathrm{pH}$ and agitation speed. The VIs are created to display the process variables, set the control parameters and to indicate the resulting control actions of the process environment. The basic function of the controller is to execute an algorithm based on the control inputs (coefficients), the desired operating value (set point) and the current process value. One of the generic control strategies is the PID (Proportional, Integral \& Derivative) control algorithms are employed to control the process parameters in industries and in many advanced control algorithms [9].

The block diagram shown in Figure 1 describes the structure of the control loop for process control operations, which is also referred as single input single output (SISO) negative feedback system [14]. The difference between the process variable and the set value is called error, which is processed by the control function with suitably tuned coefficients to maintain the error close to zero. If the error is not zero, the controller makes proper adjustment by manipulating the actuator. Transducer senses the process output and converts to an equivalent electrical quantity to form the process variable at the summing point. The timing specifications and number of samples to be processed in each control simulation are chosen based on the control parameters of the plant.

The user interface is built in LabVIEW using set of tools and objects. The user interface is known as front panel. The front panel is built with controls and indicators, which are the interactive input and output terminals of the VI, respectively. Controls are knobs, push buttons, dials and other input devices. Indicators are graphs, LEDs and other displays. Controls simulate instrument input devices and supply data to the block diagram of the VI. Indicators simulate instrument output devices and display data the block diagram acquires or generates. The graphical representations of functions form the code to control the front panel objects, which is known as block diagram. The 


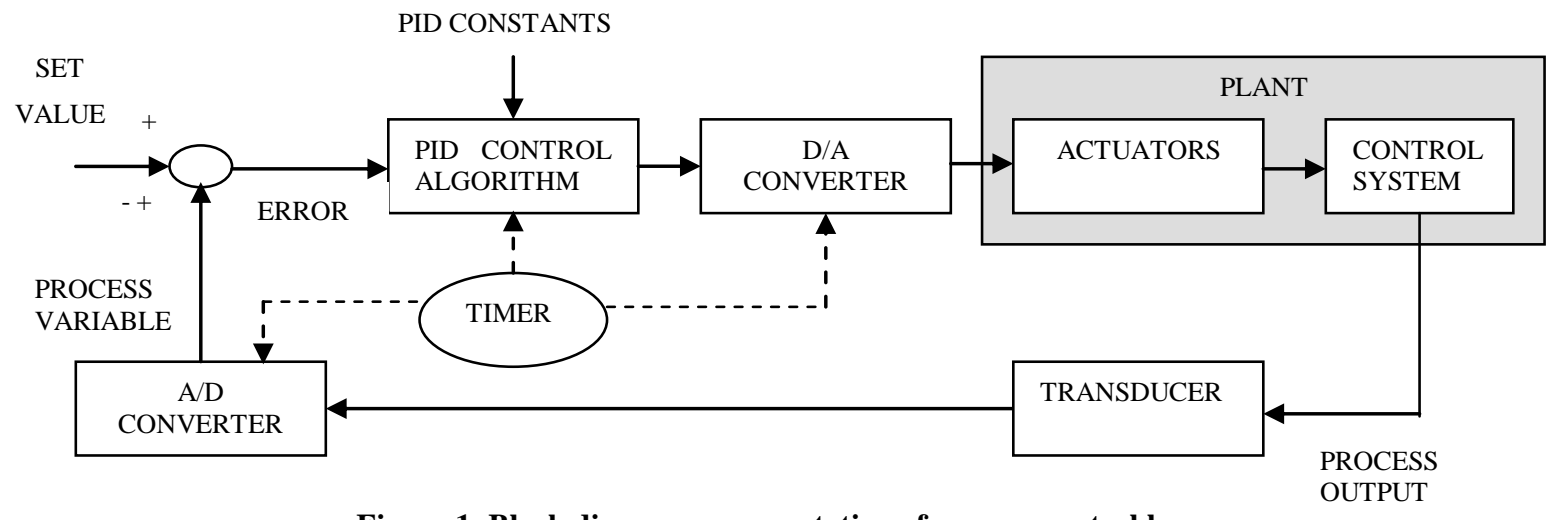

Figure 1 Block diagram representation of process control loop

block diagram contains the graphical source code. Front panel objects appear as terminals on the block diagram. The terminals are entry and exit ports that exchange information between the front panel and block diagram [16]. The independent control algorithms for each process parameters are described in the following sections.

\section{Temperature Control}

The temperature control system is designed using PID control algorithms and the system model is described in equation (3). The liquid temperature is sensed and compared it with the desired temperature to form the error signal. The error signal is processed using control algorithms to produce the desired output to the heater driver. The feed back control system operates on the heating system to maintain the temperature at the required set value by reducing the error towards zero. Continuously variable controllers are designed to produce power to the heater proportional to the error signal. As the measured value approaches its desired value, the power fed to the heater progressively reduced. PID controllers are most commonly used controllers in temperature control.

The tuning parameters are calculated using Ziegler-Nichols algorithm based on open loop and closed loop tests on system transfer functions [14]. Referring to the system transfer function described in equation (3), the PID coefficients are calculated using Ziegler-Nichols rule based on the step response of plant.

The proportional constant $K_{p}=\frac{1.2 T_{i}}{K T_{d}}=0.184$

The integral constant $T_{I}=2 T_{d}=60$

The derivative constant $T_{D}=0.5 T_{d}=15$

The controller transfer function is given by,

$U(s)=K_{p}\left(1+\frac{1}{T_{I} s}+T_{D} s\right)$
Substituting the values of tuned coefficients, equation (6) becomes,

$U(s)=0.184\left(1+\frac{1}{60 s}+15 s\right)$

The PID control simulation using LabVIEW for the temperature control environment with the known transfer function model is described in Figure 2. The input to the temperature controller is the heat flow and the output is the temperature of the liquid. The controller model is the mathematical algorithm that supplies an input to the system model, $\mathrm{H}(\mathrm{s})$ based on error. The integrator $(1 / \mathrm{s})$ follows $\mathrm{H}(\mathrm{s})$ produces the output of the plant to have consistent with set point. Scaling is performed on the set value and the output value fed to the thermometer is based on the temperature sensor output.

Assuming the temperature sensor provides $10 \mathrm{mV} /{ }^{\circ} \mathrm{C}$ produces the analog output $\mathrm{Vo}_{\mathrm{O}}=\mathrm{T} / 100$, where $\mathrm{Vo}_{\mathrm{o}}$ is the sensor output voltage in Volts, $\mathrm{T}$ is the temperature in ${ }^{\circ} \mathrm{C}$. Hence the scaling factors 0.01 are selected at the input and 100 at the output to display the output temperature. The PID output is chosen to generate the control commands to switch On/Off the heater if the error exceeds the predefined value which is decided based on the temperature tolerance level of the system. The actuating signal for the cooler is derived using the reverse logic of heating control system.

One of the practical problems with the PID controller is the "integral windup", which cause long periods of overshoot in the controller response. When the error signal is integrated for long periods of time causes an overflow, hence its value is limited to maximum and minimum levels using "In Range and Coerce" function of VI. Another possible problem in derivative action is, when the set point changes sharply, causing the error signal to give "kick" in the output, known as "derivative kick". This is avoided in practice by moving the derivative term to the feedback loop. The proportional term may also cause a sudden rise in the output and it is also common to move to the feedback loop [13]. Figure 2 shows the PID controller realization in practice. 


\section{pH Control}

In real life, bioreactors actually use on-off control for $\mathrm{pH}$ [14]. The two position control system is designed; so that the element controlling reagent addition is always set in one of two positions, either fully open or fully closed. The important consideration in

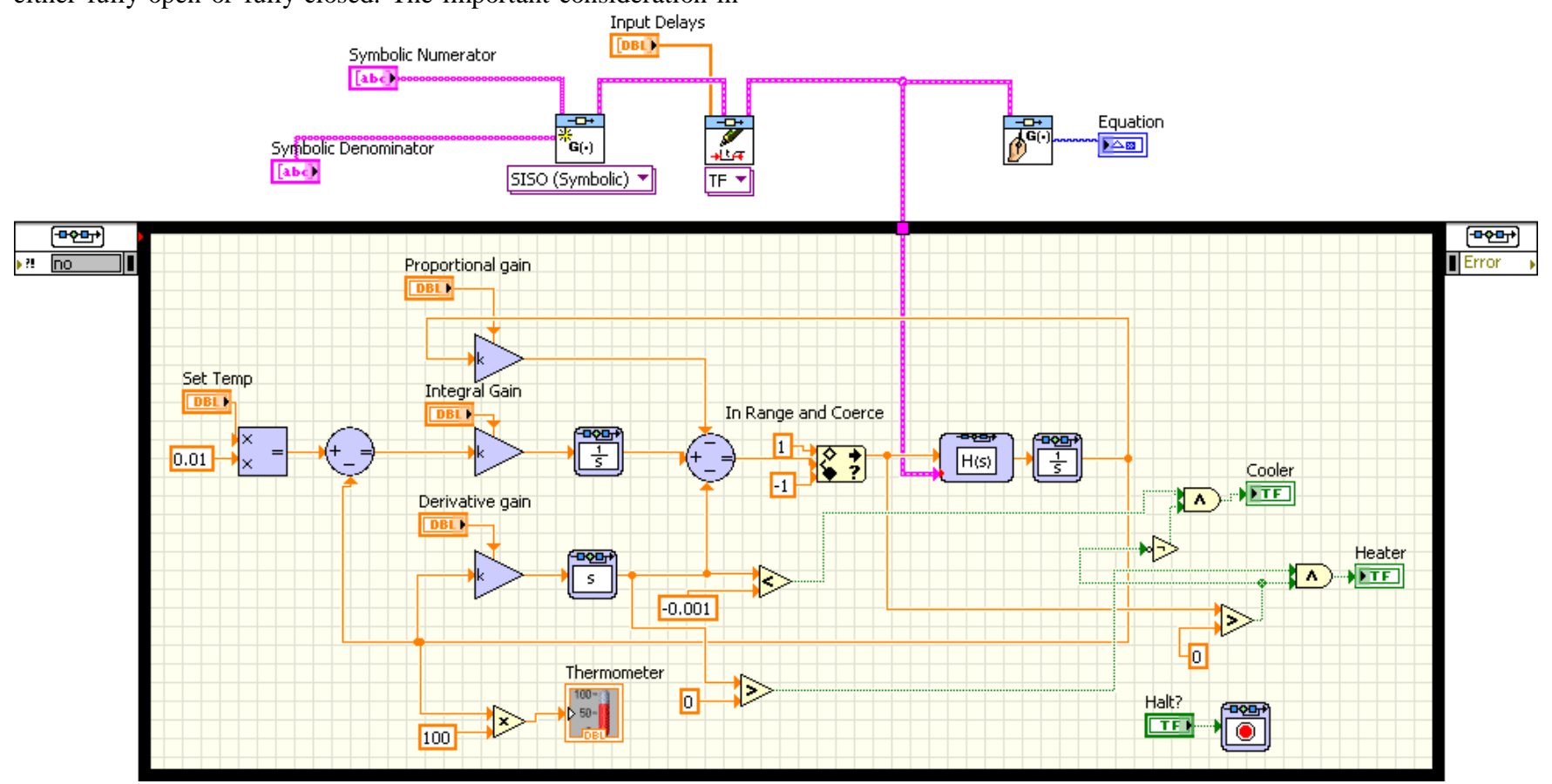

Figure 2 Graphical Code for temperature control simulation

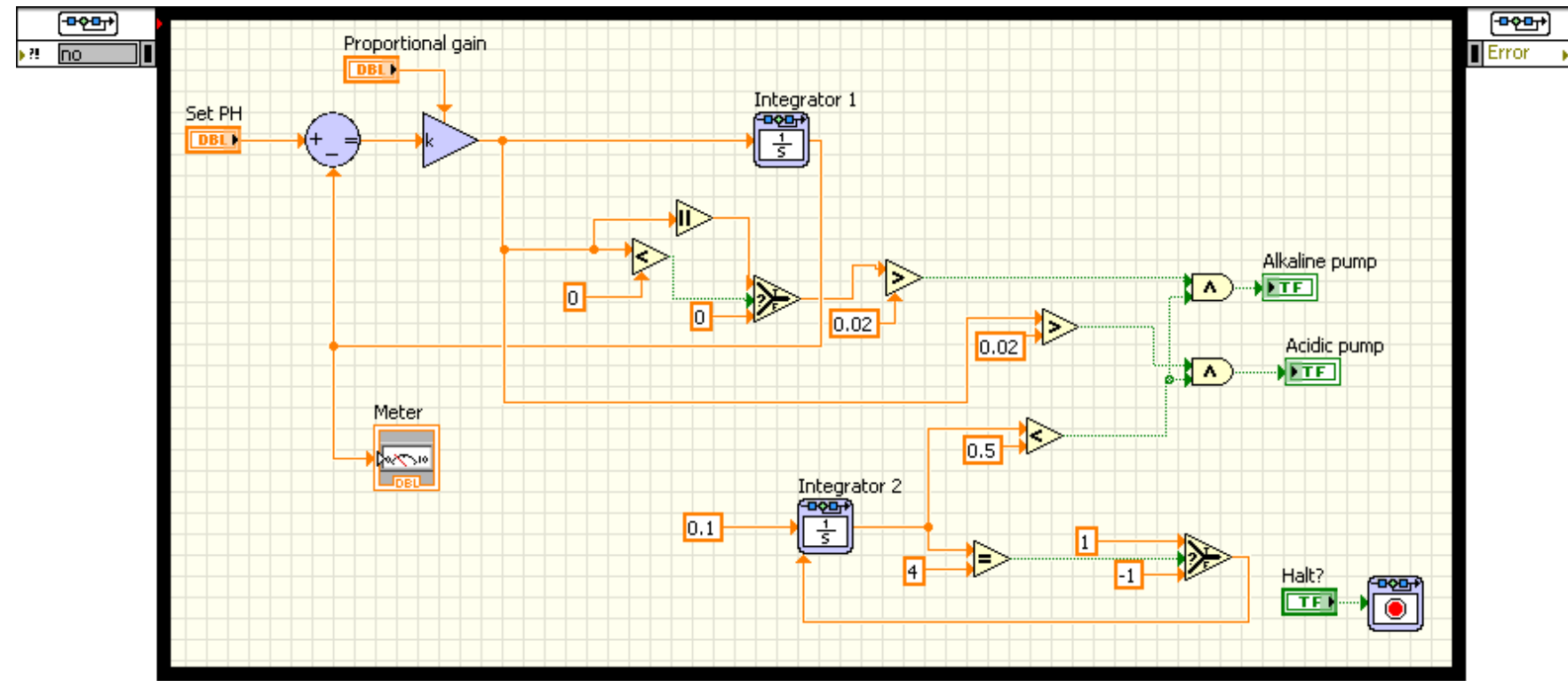

Figure 3 Graphical Code for $\mathrm{pH}$ control simulation

Based on the polarity of the proportional output the control signals are generated to the switch On/Off the Alkaline or Acid pumps with a predefined hold time. The Integrator- 2 function is used to set the hold time between the successive additions of reagents. The simulation loop is designed for hold time of 5.8
$\mathrm{pH}$ control is hold time, which is required to provide time for neutralization reaction to go to completion. This process is controlled using VI tools with simple proportional control to generate control actuations for reagent addition is shown in Figure 3. 
execution time is approximately $5 \mathrm{msec}$. The Integrator-2 produces output of 0.00005 with input 0.1 and the upper limit is 4. Integrator- 2 is reset to 0 once it reaches the upper limit. It approximately takes $6.66 \mathrm{~min}$ to reach the upper limit 4 . The control signal to the actuator is allowed only for the duration of $0.8 \mathrm{~min}$, i.e integrator count from 0 to 0.5 . The proportional output is chosen to generate the control commands, if the error exceeds the predefined value which is decided based on the tolerance level of the system.

\section{Control of Agitation Speed}

The permanent magnet DC motor described in equation (5) is used in the agitation speed control loop of the process system. The tuning coefficients are calculated using Ziegler-Nichols $2^{\text {nd }}$ method, where the system is forced to generate sustained oscillations using proportional controller and the tuning coefficients are calculated using the period of sustained oscillations and the proportionality constant [13]. The system described in equation (5) generates sustained oscillations with period $\mathrm{P}_{\mathrm{u}}=13 \mathrm{~s}$ for the proportionality constant referred as ultimate gain $\mathrm{K}_{\mathrm{u}}=0.03$. The control loop of graphical simulation is shown in Figure 4.

The PID constants and the corresponding controller transfer function given by,

$K_{P}=0.6 K_{u}=0.018$

$T_{I}=0.5 P_{u}=6.5$

$T_{D}=0.125 P_{u}=1.625$

$U(s)=0.018\left(1+\frac{1}{6.5 s}+1.625 s\right)$

The structure of the PID controller is modified to eliminate the set point kick by operating the derivative action only in the feedback. The pulse width modulated signal is simulated with its duty cycle proportional to the controller output and can be used to drive the DC motor with real-time simulations. The data in the simulation loop is suitably scaled at various levels to meet the $\mathrm{I} / \mathrm{O}$ requirements of the system. For the designed values in Figure 4 , the motor speed is controlled between 100-1000 rpm and the corresponding duty cycle of the PWM signal varied from $9 \%$ to $88 \%$.

\section{Graphical User Interface for Control and Measurements}

The control algorithm for different process parameters are combined in a simulation loop and the corresponding graphical user interface (GUI) is shown in Figure 5. The control terminals and indicators are suitably chosen to reflect their functionality in the system and also to make the operations more users friendly. The system parameters are described as transfer function coefficients and the corresponding transfer function is displayed on the front panel. The control method employed for $\mathrm{pH}$ is of OnOff control, hence only the proportional coefficient is defined on GUI. The PID tuning coefficients are set for temperature and DC motor control using vertical slide classic control terminal. The control parameters are set with in the range of control and observe the corresponding control actions on indicators on the front panel. The meters display the current process values derived from the system transfer function. The PWM signal is derived from the PID motor controller algorithm and displayed on waveform indicator on the front panel. The control signals to the actuators are derived from the controller output and can be further extended to control the actual hardware with proper driving circuits.

The controller can be optimized by the complete understanding of the plant. Thus the simulated module can be used to validate the parameters under real world constraints. The simulation parameters can be programmatically defined or the time signal can also be derived with the external hardware. Once a control system is designed with the control and simulation module; the algorithm can be deployed to control a real physical system. The deployed algorithm can be run on desktop PC or real-time systems. Running a control system on desktop PC is possible by simply replacing the plant model with the hardware input and output functions. Data acquisition tasks are configured with timing parameters, so it is important to equate the simulation loop with the data acquisition timing. 


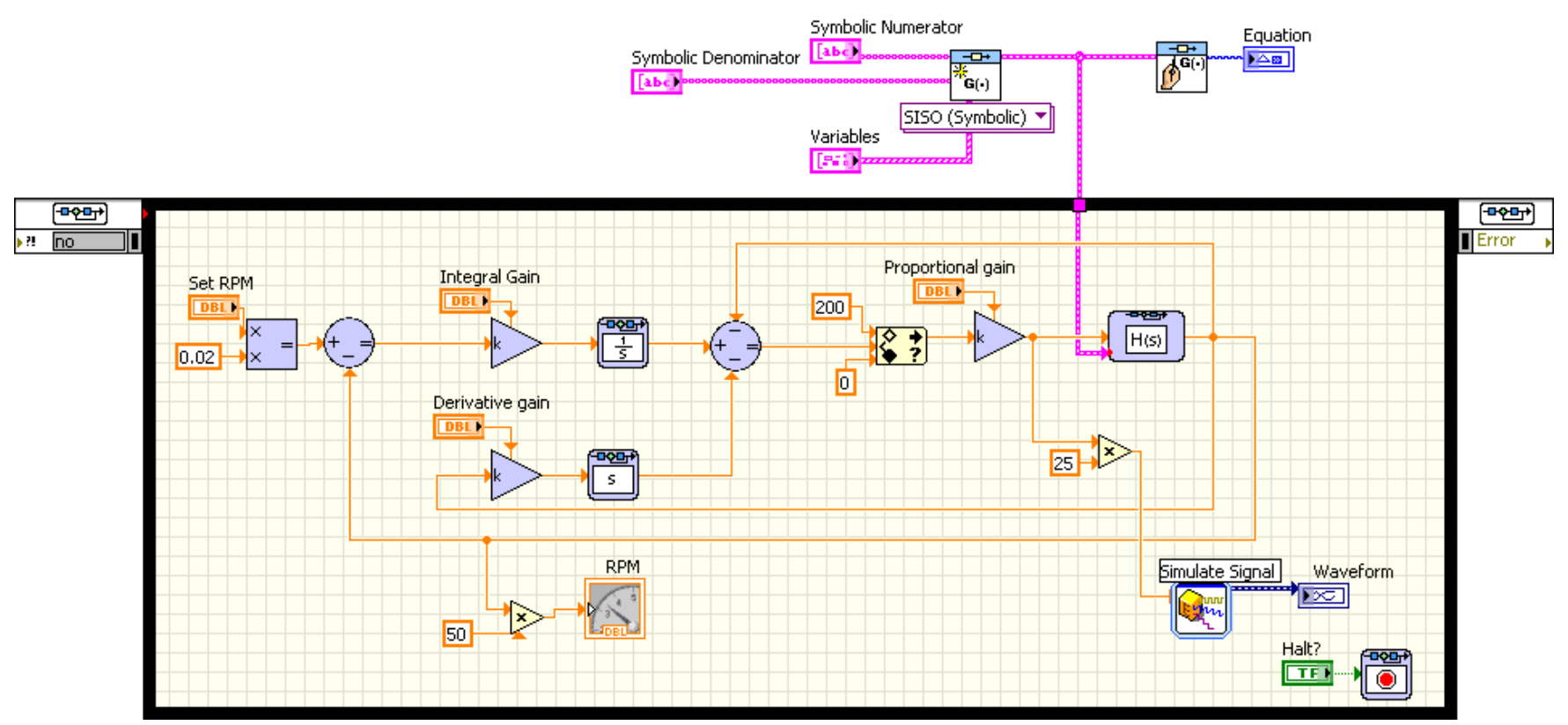

Figure 4 Graphical Code for DC motor control simulation

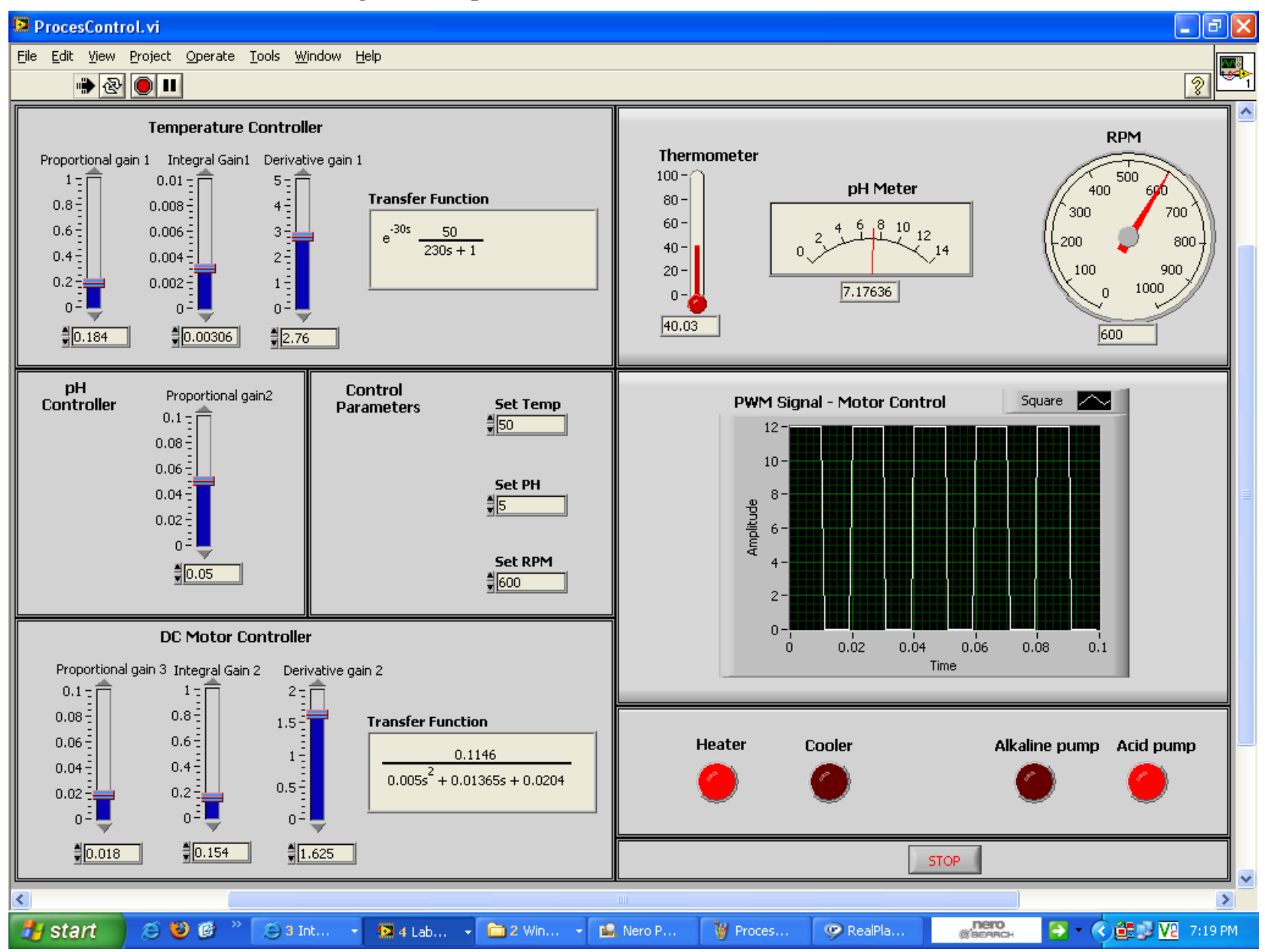

Figure 5 Graphical User Interface for process control simulation 


\section{HARDWARE INTERFACE AND REMOTE ACCESS FEATURES}

Virtual instrumentation combines hardware and software with industry-standard computer technologies to create solutions for user-defined applications. Data Acquisition system acts as the interface between the computer and the outside world. The design elements of graphical code terminals and indicators can be easily interfaced to real hardware through the data acquisition system. The remote access and control interface structure for bioreactors control parameters are shown in Figure 6.

LabVIEW Web Server is used to create HTML documents, publish front panel images and embed VIs in a web page. The VI front panel can be viewed remotely, either from within LabVIEW or from within a web browser, by connecting to the LabVIEW built-in web server. When the front panel remotely opened through the web, the web server sends the front panel to the client, but the block diagram and all other subVIs remain on the

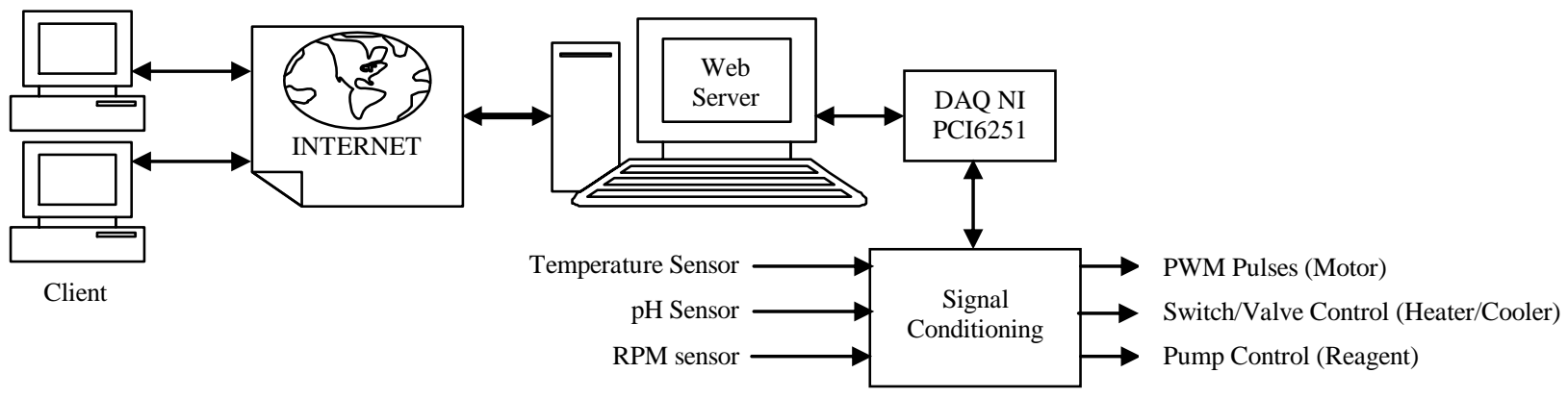

Figure 6 Remote Access Structure of process control parameters

server computer. The remote client can interact with the front panel in the same way as if the VI were running on the client, except the block diagram executes on the server. This feature is used to publish entire front panel or to control the remote application safely and easily [16]. The control loop is installed on server and the control actions are regenerated whenever the clients updates the changes in control parameters on web page. The old sets of parameters are active until a new set of parameters are received by the web server. Therefore, the internet delays do not affect the system performance. At the client side, LabVIEW run-time engine has to be installed to access front panel through web page.

The National Instruments DAQ card PCI6251 supports 2 channels of analog outputs, 12 channels of analog inputs, 8 channels of independent digital I/Os and two counter outputs. The interface levels of data acquisition system are $\pm 10 \mathrm{~V}$ for analog and digital signals are TTL compatible. The analog inputs of DAQ are mainly interfaced with the sensors outputs corresponding to temperature, $\mathrm{pH}$ and motor speed. The signal conditioning unit is the electronic circuitry which interfaces the physical components of the process with the data acquisition system. The control signals to the actuators to operate the switch/valve/pump to control heater/cooler/reagent can be derived using digital outputs applied to the suitable driving circuitries of the actual hardware. Similarly the PWM pulses to control the DC agitation speed is available at the analog output of DAQ. The data acquisition and processing operations are performed by acquiring finite number of samples and post processing them to generate the actuations to the system.

\section{Web Based Tools for Remote Experiments}

The remote access and control of bioreactor parameters is possible through LabVIEW built-in web server. But, it is necessary to create web-learning environment and to provide multimedia interface to extend its operation to remote experimentation. The integration of collaborative tools with the remote experimentation provides opportunities to interact, practice and to understand the need for working.

Universities are using Web 2.0 technology tools are collectively referred as social software, where in teachers, researchers and students started to use for remote learning. Learning Management System (LMS) is responsible for educational interactions at the institutional level by integrating geographically dispersed resources. The integration of remote experimental system with the LMS tools gives more scope to distance learning of science and engineering domains. The majority of the courses in these domains are supported by practical laboratory activities. It is also possible to integrate the LMS tool such as Moodle (Modular Object Oriented Dynamic Learning Environment) to provide learning resources, web camera streaming software WebcamXP to broadcast audio and video features, and LabVIEW to make user interaction with the distant system, to develop collaborative remote experiment systems [4]. LMS tools support collaborative learning in remote laboratory and distributed workspaces. The webcamXP software streams live videos on to client's machine without any additional programming. Combining LMS tools with LabVIEW for collaborative learning enables the design of a versatile educational environment for science and engineering.

\section{CONCLUSION}

The use of VI in simulation and control of bioreactors environment parameters and the collaborative features to extend its operation to remote experimentation are described. The system models are designed by considering the real time scenarios and are integrated to obtain combined system response for bioreactors simulation. The control algorithms simulated can be extended to operate the physical systems by synchronizing the timing with the hardware module. 
The designed system can be used independently with the described experiment and it can also be combined with similar industrial control applications. Besides using it in the education, it is also possible to develop and improve the controller designs in industries. PID is a generally applicable control technique for process applications that drives its success from simple and easyto understand operation. Many design methods can be automated with simulation packages and the trend of computerizing the control operations are gaining momentum. The design uses the standard PID structure by automating the tuning process with the modular code blocks made available for real-time applications.

\section{ACKNOWLEDGMENTS}

This paper reports the partial results of a major research on Remote Access Labs funded by the Department of Science and Technology, Ministry of Science and Technology, Government of India, Vide Number: SR/FST/ETI-033/2005.

\section{REFERENCES}

[1] Alia, M.A.K. and Zalata, M.K.A. 2004. "A closed loop temperature control system by utilizing a LabVIEW customdesign PID controller", 7th United Kingdom simulation society conference, Oxford, England.

[2] Aung, W.P. 2007. Analysis of Modeling and Simulink of DC Motor and its Driving System used for wheeled Mobile Robot. World Academy of Science, Engineering and Technology. Vol. 32, 299-306.

[3] Chandra, J.A.P. and Samuel, R.D.S. 2009. Design of novel online access and control interface for remote experiment on DC drives. International Journal of Online Engineering Vol.5, No. 2, 11-17.

[4] Chandra, J.A.P. and Samuel, R.D.S. 2009. Internet Accessed Collaborative Learning Solutions for Remote Experiments using Web 2.0 Social Software and LabVIEW. International Conference on Software Technology and Engineering, Chennai, India, 146-150.

[5] Cvjetkovic, V.M., Matijevic, M. and Stefanovic, M. 2008. Universal Web development system for implementation of controllers for remote real systems. International Conference on Remote Engineering and Virtual Instrumentation. Duesseldorf, Germany.

[6] Gilibert, M., Picazo, J., Auer, M., Pester, A., Cusido, J. and Ortega, J.A. 2006. 80C537 Microcontroller Remote Lab for E-Learning Teaching. International Journal of Online Engineering. Vol. 2, No. 4, 1-3.

[7] Junior, C.A.V., Costa, C.T.O.G., Carreiro, A.M.C. and Araujo, O.Q.F. 2005. Internet-Based Process Operation: An Educational Approach. 2nd Mercosur Congress on Process Systems Engineering, Rio de Janeiro, Brazil, 1-8.

[8] Li, S. And Khan, A.A. 2005. Applying IT tools to a laboratory course for measurement, analysis and design of electric and electronic circuits. IEEE Transactions on Education, Vol.48, No.3, 520- 530.

[9] Li, Y., Ang, K.H. and Chong, G.C.Y. 2006. PID Control System Analysis and Design, Problems, Remedies, and Future Directions. IEEE Control Systems Magazine (February 2006). 32-41.

[10] Muller. D., Bruns, F.M., Erbe, H., Robben, B. and Yoo, Y. 2007. Mixed reality learning spaces for collaborative experimentation: A challenge for engineering education and training. International Journal of Online Engineering. Vol.3, No.4, 15-19.

[11] Naumovic, M.B. and Zivanovic, D. 2008. Remote Experiments in Control Engineering Education Laboratory. International Journal of Online Engineering Vol.5, No. 2, 273-282.

[12] Thomas E. Kissell. Industrial Electronics, 3rd Edition. Prentice Hall of India, New Delhi. 2003.

[13] Dogan Ibrahim. Microcontroller based Temperature Monitoring and Control. Elsevier Science and Technology Books. 2002.

[14] Pao C. Chau. Chemical Process Control: A First Course with MATLAB. 2001.

[15] LabVIEW Control Design Toolkit user manual. National Corporation Instruments. 2007.

[16] LabVIEW 7 Express User Manual. National Instruments Corporation. 2003. 